Estudios Geológicos, 67(2)

julio-diciembre 2011, 171-176

ISSN: 0367-0449

\title{
Léonard Ginsburg et la Paléontologie Portugaise. Hommage amical
}

\section{Léonard Ginsburg and the Portuguese Palaeontology. A friendly tribute}

\author{
M. Telles Antunes ${ }^{1,2}$
}

\section{EXTENDED ABSTRACT}

Léonard Ginsburg has been a distinguished palaeontologist. He was a gifted one: intelligence, high working capacity, experience, knowledge and critical sense. As critical sense is not always the best for some people, adversaries were not rare. He was both an accomplished researcher and a deep connoisseur of the collections under his responsibility, as well as someone with a broad knowledge of the science world. By the high number and value of his contributions, an homage that he deserved so much is but an act of justice.

As far as I am concerned, Ginsburg was a Colleague and Friend since 1961 until the end, even after his research activities ceased. Of course, his contributions cannot be valued just from a personal viewpoint. We can recognize their value for, among many others, a Country as Portugal.

After obtaining a scholarship to prepare my Ph.D., I drove to Paris loaded with vertebrate fossils from Angola and Portugal, not without a few incidents because, by sheer chance, I arrived just the day of the Algerian "putsch". Here they are my beginnings in Paris that developed into an institutional and personal collaboration and friendship for half a century.

I have been very well received by the Director of the Muséum's Institut de Paléontologie, Professor Jean-Pierre Lehman. I was lodged at the building's basement. Soon afterwards, an unknown man approached asking me if I was a young «Spaniard 》 that came in to study fossil vertebrates, as Mr. Lehman had told him. He was Léonard Ginsburg. Our collaboration begun.

We dealt mainly with the Miocene mammalian faunas from Portugal, and especially from Lisbon, as well as some Eocene ones. Nevertheless we also studied some other groups as crocodilians and areas, mostly in France. Research was carried on at the Paris Museum, the concerned Portuguese institutions and on the field.

Under a palaeontological viewpoint, a most important place concerns Carnivora and Rhinocerotidae, as well as palaeoecologic and stratigraphic consequences. Some new taxa were described. Studies on taphonomic matters were produced.

New interpretations taking profit of the very favourable conditions presented by the Tagus basin in Portugal were most useful for marine-continental correlations. Results may in part be recognised through the list of briefly commented papers given in the French text. It comprises both the papers signed by him as co-author and other ones where smaller contributions are recorded. The two ones dated 2006 are perhaps his last papers.

As Ginsburg was compelled to quit the Muséum, he lost indispensable means of work. Research had to cease. This undoubtedly accelerated his physical decadence.

In my albeit modest opinion, Ginsburg largely deserves the recognition from the Institution that he served for so long in such a remarkable way and from all those with which he collaborated, or that profited from his help.

Otherwise, we clearly can conclude that he gave (among many others) a most valuable contribution to the Palaeontology and Stratigraphy of the Portuguese Tertiary. This justified his election as a Foreign Corresponding Member of the Lisbon Academy of Sciences.

As a memory of his work, his collaboration and friendship, I want to express here my deep gratitude and sympathy towards my dear Friend, Léonard.

Our common Friend, Philippe Taquet, had the touching courtesy of communicating me the death of Ginsburg and to put a flower on my intention on his grave at burial.

REQUIESCAT IN PACE!

\footnotetext{
1 Academia das Ciências de Lisboa, R. da Academia das Ciências, 19, 1249-122 Lisboa, Portugal.

Email: migueltellesantunes@gmail.com.

2 Centro de Investigação em Ciência e em Engenharia Geológica da UNL, Campus de Monte de Caparica, Portugal.
} 


\section{Avant propos}

Léonard Ginsburg fut un paléontologue remarquable, doué de grandes qualités d'intelligence, d'une capacité de travail, d'un savoir et d'une expérience rares, possédant, en outre, un sens critique qui lui valut nombre de contradicteurs.

Très au fait du monde scientifique, il était à la fois un chercheur accompli et un profond connaisseur des collections dont il avait la responsabilité.

Il fut, en ce qui me concerne, un fidèle compagnon de route dès 1961 et jusqu'à la fin de ses activités dont l'arrêt signifia pour lui, plus que probablement, le préalable à la mort.

Un témoignage personnel ne peut, seul, rendre compte de l'ampleur de ses contributions scientifiques mais il permet néanmoins de faire reconnaître leur valeur en ce qui concerne un pays comme le Portugal.

Le texte qui suit résume l'histoire de notre rencontre et de notre collaboration.

\section{Débuts}

Assistant depuis peu à la Faculté des Sciences de Lisbonne, je quittai cette ville le mardi 18 Avril 1961 à bord d'une « coccinelle » Volkswagen remplie de vertébrés fossiles de l'Angola et du Portugal. Mon principal but était la préparation, à Paris, d'une thèse de Doctorat sur les vertébrés du Crétacé et du Tertiaire de l'Angola. Un autre objectif, non moins important, résidait dans l'étude de mes récoltes concernant principalement le Miocène à Lisbonne. Une bourse d'études m'avait été attribuée par la Fondation Calouste Gulbenkian.

Le choix du Muséum national d'Histoire naturelle avait pour origine un courrier me concernant adressé par le Professeur Carlos Teixeira (19101982) à son Collègue français Jean Piveteau (18991991). A l'Institut de Paléontologie du Muséum se trouvaient les collections qui m'intéressaient. De plus, on venait de construire le " nouveau bâtiment » élargissant l'espace disponible et aidant de nouvelles possibilités pour la recherche et pour les chercheurs.

Le voyage ne se déroula pas sans incidents et ce, dès la frontière Espagnole de Fuentes de Oñoro. Un véhicule bien rempli, avec, comme seul passager, le conducteur, ne manque pas de faire naître des soupçons quant à la nature de son chargement ... drogue, matériel subversif ! Un fonctionnaire de la
Guardia Civil, coiffé du célèbre couvre-chef de cuir noir m'interpella et me fit ouvrir l'un de mes nombreux paquets. A l'intérieur se trouvaient des os de plésiosaures angolais. "Huesos ! son los huesos del Galvón ! » et il éclata de rire. [Henrique Galvão: révolutionnaire qui avait détourné peu avant le paquebot portugais Santa Maria].

A la fin de ma première étape, j'arrivai à Salamanca dans la lumière magnifique de la fin d'après midi. Je passai par le pont Romain, profitant au maximum de la «belle vue ». Malheureusement, j'ignorai que la circulation automobile était interdite dans cette partie de la ville, - ce que rien ne précisait -. En conséquence, la « belle vue » me valut une amende !

J'arrivai à Paris en fin de journée après avoir fait un détour par Chartres, détour qui me permit d'admirer la cathédrale, cette merveille de l'Art Gothique, tout comme le jour précédent, j'avais fait halte à Burgos.

Peu avant Chartres, j'ai du stopper devant un barrage tenu par les CRS (Compagnie Républicaine de Sécurité). Là, contrôle d'Identité, des documents de la voiture ... Peu après Chartres, nouveau contrôle par des éléments fortement armés: une mitrailleuse en batterie derrière une barrière métallique « couvrait » mon véhicule à la hauteur de ma tête. Un fonctionnaire contrôle mes documents et s'enquiert du contenu d'un certain paquet triangulaire situé derrière moi: « une mitraillette ? » il s'agissait du crâne d'un crocodile jurassique ! Je m'interrogeai sur les raisons de ces contrôles réitérés dans un pays dit démocratique, moi, qui venait de traverser des pays « totalitaires ».

C'est en arrivant au petit Hôtel de la Rue de l'École de Médecine où l'on m'avait réservé une chambre que les deux collègues qui m'attendaient, m'apprirent les raisons de ces mesures surprenantes. Nous étions le Vendredi 21 Avril le jour même du « putsch d'Alger ». J'avais déjà remarqué, en suivant dans ma « coccinelle » le taxi qui transportait ma valise, le peu de trafic dans les rues de la capitale. $\mathrm{Si}$, plus tard, accompagné de mes collègues, je ne rencontrai, de nouveau, que peu de voitures, je constatai en revanche la présence de forces militaires - y compris des tanks - dans les lieux stratégiques comme la Place de la Concorde. Quel privilège bizarre d'être là, présent, à ce moment donné de l'Histoire de la France ! On craignait l'arrivée de parachutistes ... qui finalement ne virent jamais.

Le lendemain, j'assistai, en « curieux », à une manifestation (évènement alors interdit au Portugal) 


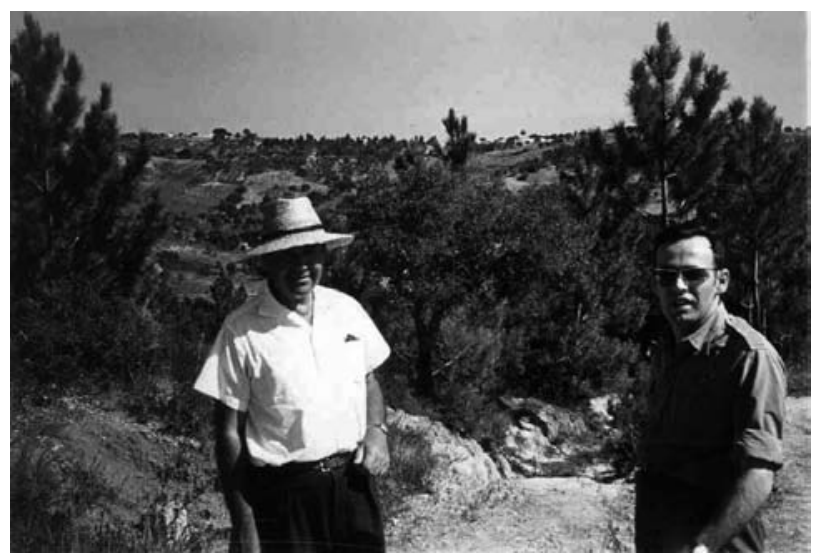

Fig. 1.-Georges Zbyszewski et Miguel Telles Antunes à Aveiras de Baixo (Ribatejo, Portugal) en Octobre 1963. Photo L. Ginsburg.

Boulevard St Michel. Y figuraient des jeunes et des moins jeunes. Tous scandaient des slogans sous équivoque «Le fascisme ne passera pas » etc.

Tels furent mes débuts en France, à Paris, débuts qui inauguraient d'une manière imprévue et pour le moins originale une période de près de cinquante ans de collaboration institutionnelle et personnelle avec les collègues de ce pays et au cours de laquelle je novais de solides amitiés.

\section{Développements}

Je me présentai, peu de temps après à l'Institut de Paléontologie où je fus reçu très aimablement par son Directeur le regretté Jean-Pierre Lehman (19141981). Son appui et ses conseils me furent très précieux. Il alla même plus loin quelques années plus tard, me faisant la généreuse proposition d'intégrer, en qualité de chercheur, le Centre National de la Recherche Scientifique afin de m'éviter une mobilisation lors de la guerre que menait le Portugal en Afrique. Outre la possibilité de faire une carrière qui m'intéressait dans les meilleures conditions, cela me délivrait d'un véritable cauchemar. Cela impliquait, toutefois, un changement de Nationalité et faisait de moi, d'une certaine façon, un déserteur ! Aussi préférai-je courir le risque de partir pour la guerre et déclinai sa proposition mais je lui ai gardé une énorme dette de reconnaissance.

On me logea au sous-sol où je pus étaler mon matériel et commencer à organiser mon travail. Et c'est là, qu'un après-midi, un inconnu s'approche de moi et me demande si j'étais bien ce « jeune

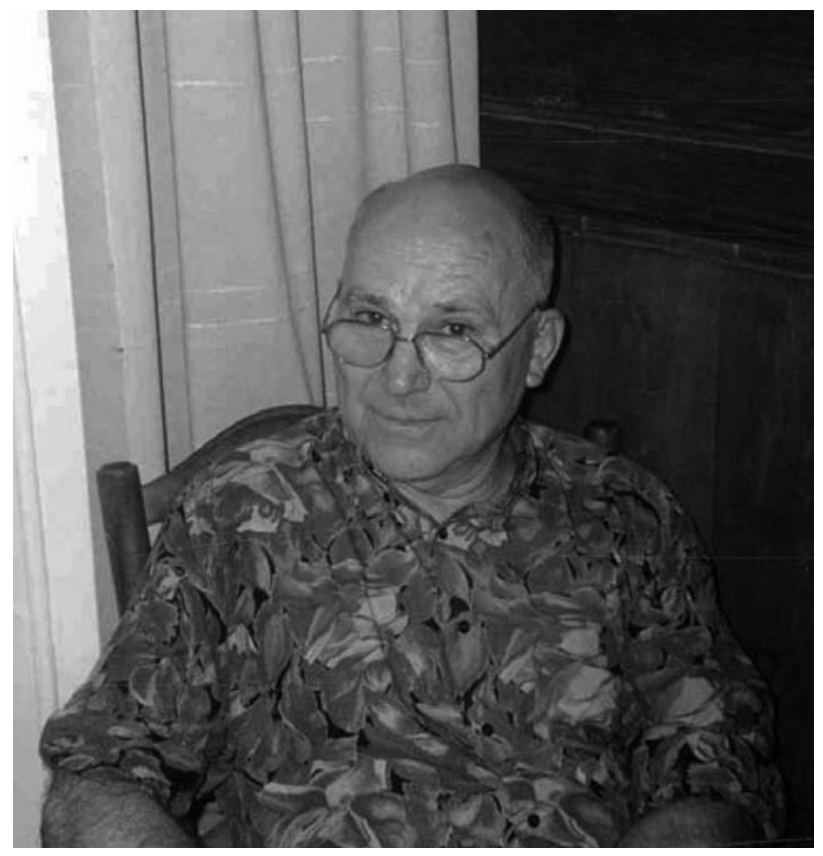

Fig. 2.-Léonard Ginsburg chez lui, un appartement dans un ancien couvent du $17^{\text {ème }}$ au Quartier du Marais, Rue Jarente, Paris IVe. Juillet 1998. Photo M.T. Antunes.

espagnol »venu étudier les vertébrés fossiles et dont M. Lehman lui avait parlé ... c'était Léonard Ginsburg. Ainsi débuta une collaboration qui devait durer un demi siècle !

Cette collaboration concerna principalement les faunes de mammifères du Miocène portugais et particulièrement celles de Lisbonne, en plus de celles de l'Eocène du même Pays. Nous ne laissâmes toutefois pas de côté d'autres groupes - dont les crocodiliens - et d'autres régions, en France notamment.

Si l'essentiel de ces activités eut lieu au Muséum presque chaque année, Ginsburg eut aussi l'occasion de se déplacer au Portugal ayant accès aux collections des institutions concernées et visitant les gisements avec moi et avec des Collègues dont Octávio da Veiga Ferreira (1917-1997) et Georges Zbyszewski (1909-1999).

Je me dois, de plus, de lui témoigner ici, post mortem, ma gratitude. En effet, au cours de la préparation de ma thèse il me fut indispensable de me rendre à Londres, Bruxelles et Tervuren ... et cela sans soutien financier ! La bourse était insuffisante. Malgré une épargne sévère, mes ressources s'épuisèrent. Ce fut alas Ginsburg qui, en m'embauchant pour fouiller, malgré la neige, ses chers faluns de Touraine, sauva la situation. 


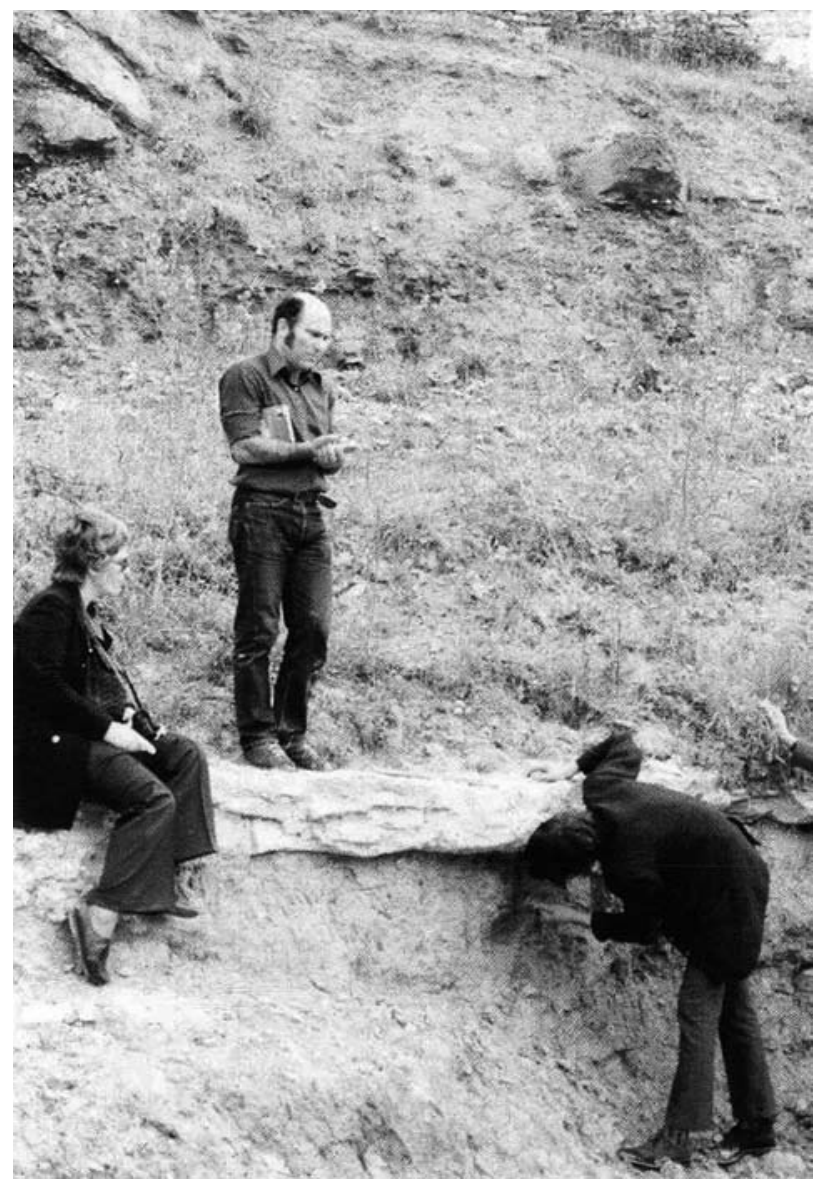

Fig. 3.-L. Ginsburg en train de présenter (le 11 Juin 1973) son très cher gisement de Sansan (Gers) au cours d'une excursion après le Colloque International du CNRS $N^{\circ} 218$ - Problèmes actuels de Paléontologie (Évolution des Vertébrés), Paris 4-9 Juin. Photo M.T. Antunes.

\section{Quelques résultats}

Sur le plan paléontologique, on peut reconnaître la partie spécialement importante qui revient à l'étude des mammifères, carnivores et rhinocérotidés surtout, y comprises les interprétations paléoécologiques et stratigraphiques. Quelques taxa nouveaux furent décrits. Nous menâmes finalement à bien des études sur des thèmes de taphonomie: ces résultats comptent même parmi les derniers avec intervention de Léonard Ginsburg.

Les taxa suivants furent considérés comme nouveaux lors de leur description :

Equidés: Paranchilophus lusitanicus (Ginsburg, 1965), Eocène ;

Carnivores: Amphicyon olisiponensis Antunes \& Ginsburg, 1972, carnivore amphicyonidé du Miocène inférieur; Plithocyon antunesi Ginsburg \& Morales,
1995, ursidé du Miocène inférieur et moyen; Hemicyon sansaniensis parvus n. ssp. Ginsburg \& Antunes, 1995, un autre ursidé du Miocène moyen.

Rhinocérotidés: Chilotherium ibericus Antunes, 1972; Aceratherium lumiarense Antunes \& Ginsburg, 1983; Gaindatherium (Iberotherium) rexmanueli Antunes \& Ginsburg, 1983; Gaindatherium (Iberotherium) rexmanueli ssp. zbyszewskii Antunes \& Ginsburg, 1983.

De nouvelles interprétations ont eu des conséquences pour des corrélations entre unités marines et continentales, profitant en spécial des conditions particulièrement favorables du Bassin du Bas Tage au Portugal.

On peut se rendre compte de divers résultats dans les publications qui suivent, comprenant celles dont Ginsburg était co-auteur et d'autres où l'on reconnaît des contributions plus limitées par lui.

\section{1}

Miguel Telles Antunes - TOMISTOMA LUSITANICA, crocodilien du Miocène du Portugal. Rev. Fac. Ciênc. Lisboa, $2^{\mathrm{a}}$ sér., C, IX (1), p.5 - 88, 12 est. [P. 75: «... L. Ginsburg, ..., a fait des corrections dans quelques chapitres et nous communiqua aussi quelques trouvailles de crocodiliens qu'il a faites dans les faluns de Noyant-sous-le-Lude».

\section{4}

Miguel Telles Antunes - Présence du genre Palaeotherium Cuv. (Equoidea, Mammalia) dans les argiles de Côja (Arganil) / Considérations sur l'âge et l'extension des formations éocènes au Portugal. Revista da Faculdade de Ciências de Lisboa, $2^{\mathrm{a}}$ série C, vol. XIII, fasc. $1^{\circ}$, p.103-122, 1 est. P.110: « Mesures ... prises par ... L. Ginsburg ...». Il s'agît de la première contribution paléontologique et datation concernant l'Eocène au Portugal.

\section{5}

Miguel Telles Antunes - Notes sur la Géologie ... V - Un Schizotheriiné du genre Phyllotillon (Chalicotherioidea, Perissodactyla) dans 1'Helvétien V-b de Charneca do Lumiar / Remarques écologiques sur la faune de mammifères. Bol. Soc. Geol. Port., vol.XVI, fasc.1, p. 159-178, 2 est. [P. 177: «L'auteur remercie vivement à MM. L. GINSBURG, M. CRUSAFONT et G. ZBYSZEWSKI toute l'aide qu'ils ont bien voulu lui accorder.»

\section{6}

L. Ginsburg \& M. Telles Antunes - Considérations sur les mastodontes du Burdigalien de Lisbonne et des sables de 1'Orléanais (France). Rev. Fac. Ciênc. Lisboa, $2^{\mathrm{a}}$ sér. C, vol. XIV, fasc. 2, p. 135-150, 4 est. On a montré que plusieurs taxa de mastodontes du Miocène tombent en synonymie.

\section{8}

L. Ginsburg \& M. Telles Antunes - Amphicyon giganteus, carnassier géant du Miocène. Annales de Paléontologie, Vertébrés, tome LIV, p. 1-32, 1 est. Masson \& Cie, Paris. 


\section{2}

M. Telles Antunes, J. Viret \& G. Zbyszewski - Notes sur la Géologie ... X- Une conférence de J. Viret sur 1'Hispanotherium (Rhinocerotidae) / Quelques données complémentaires, autochtonie et endémisme. Bol. Mus. Lab. Miner. Geol. Univ. Lisboa, 13 (1), p. 5-23, 2 est. [P. 18: Les études de M. T. Antunes et L. Ginsburg ont permis de vérifier, ..., que l'Hispanotherium était ... le rhinocéros le plus abondant dans ... l'Helvétien V- $b$ de Lisbonne].

\section{3}

M. Telles Antunes, L. Ginsburg, J. R. Torquato e M. L. Ubaldo - Âge des couches à Mammifères de la basse vallée du Tage (Portugal) et de la Loire moyenne (France). C.R. Acad. Sc. Paris, t. 277 (26 Novembre 1973), Série D, p. 2313-2316. Synthèse fondamentale: échelle stratigraphique régionale, datation d'après des foraminifères planctoniques, K-Ar, échelle continentale sur des mammifères.

\section{7}

M. Telles Antunes \& L. Ginsburg - Notes sur la Géologie ... XIX- Sur un Amphicyon (Mammalia, Ursidae) du Burdigalien. Comunic. Serv. Geol. Port., t. LXI, p. 335-342, 2 est. Reconnu pour la première fois sur du matériel de Lisbonne.

M. Telles Antunes \& P. Mein - Contributions à la Paléontologie du Miocène moyen continental du bassin du Tage / III / Mammifères- Póvoa de Santarém, Pero Filho et Chões (Secorio) / Conclusions générales. Ciências da Terra (UNL), Lisboa, $\mathrm{n}^{\circ} 3$, p. 143165, 3 est. [P. 157: «L'étude des grands mammifères a été réalisée, en partie, à l'Institut de Paléontologie ... de Paris, où Mr. L. Ginsburg a bien voulu vérifier ... l'Amphicyon de Pero Filho.»]

\section{9}

Miguel Telles Antunes - «Hispanotherium fauna» in Iberian middle Miocene, its importance and paleogeographical meaning. Annales géologiques des Pays helléniques, Hors Série, fasc. I, 1979, p. 19-26. VII th International Congress on Mediterranean Neogene, Athens 1979. [P. 25: «We are indebted to: ... Dr. L. Ginsburg, ...»].

Miguel Telles Antunes - Vertebrados miocénicos de Olhos de Água (Algarve), interesse estratigráfico. Bol. Mus. Lab. Miner. Geol. Fac. Ciênc., vol. 16 (1), p. 343-352, Lisboa, 1979. [P. 351: «... agradecimentos ... L. Ginsburg ...»].

L. Ginsburg \& M. Telles Antunes - Les Rhinocérotidés du Miocène inférieur et moyen de Lisbonne (Portugal). Succession stratigraphique et incidences paléogéographiques. C. R. Acad. Sc. Paris, t. 288 (5 Février 1979), Série D - p. 493-495. [Note préliminaire].

\section{3}

M. Telles Antunes \& L. Ginsburg - Les Rhinocérotidés du Miocène de Lisbonne - Systématique, Écologie, Paléobiogéographie, valeur stratigraphique. Ciências da Terra (UNL), Lisboa, $\mathrm{n}^{\circ} 7$, p. $17-98,16$ est.

M. Telles Antunes, L. Ginsburg e P. Mein - Mammifères miocènes de Azambujeira, niveau inférieur (Santarém, Portugal). Ciências da Terra (UNL), Lisboa, $\mathrm{n}^{\circ}$ 7, p. 161-186, 3 est.

\section{4}

Miguel Telles Antunes - Essai de synthèse sur les Mammifères du Miocène du Portugal. Volume d'hommage au géologue
G. Zbyszewski, Éd. Recherche sur les Civilisations, Paris, 1984, p. 301-323. [P. 305: «... progrès ... ont été possibles en partie ... grâce à la collaboration ... de ..., en particulier: L. Ginsburg ...»].

\section{6}

Miguel Telles Antunes - Acerca de um osso do Plistocénico da Mealhada: presença de um «tigre dente de sabre», Homotherium latidens (Owen, 1846). Ciências da Terra (UNL), Lisboa, $\mathrm{n}^{\circ}$ 8, p. 43-54, 1 est. [P. 50: L. Ginsburg ... a fourni des moulages ....].

Miguel Telles Antunes - Paralophiodon cf. leptorhynchum (Tapiroidea, Mammalia) à Vale Furado: contribution à la connaissance de 1'Eocène au Portugal. Ciências da Terra (UNL), Lisboa, $n^{\circ}$ 8, p. 87-98, 1 est. [P. 93: ... appui, ... L. Ginsburg ...].

Miguel Telles Antunes - Anoplotherium (Mammalia, Artiodactyla) et Geochelone (Reptilia, Testudines) à Côja: les vertébrés fossiles et 1'Eocène supérieur au Portugal. Ciências da Terra (UNL), Lisboa, n ${ }^{\circ} 8$, p. 99-110, 1 est. [P. 106: «... remerciements à M. L. Ginsburg ...»].

Miguel Telles Antunes - Iberosuchus et Pristichampsus, crocodiliens de 1'Eocène / données complémentaires, discussion, distribution stratigraphique. Ciências da Terra (UNL), Lisboa, $\mathrm{n}^{\circ}$ 8, p. 111-122, 1 est. [P. 118: «... remerciements ...L. Ginsburg ... facilités ..., y compris l'étude des spécimens d'Argenton décrits par Cuvier dont le type de Pristichampsus rollinati, et le prêt de spécimens de Robiac»].

M. Telles Antunes \& C. Estravís - Suidés et Tayassuidés du Burdigalien V- $a$ et du Langhien V- $b$ de Lisbonne. Ciências $d a$ Terra (UNL), Lisboa, ${ }^{\circ}$ 8, p. 139-178, 7 est. [P. 153: ... Mr. Léonard Ginsburg, ... a bien voulu mettre à notre disposition les collections ...].

\section{7}

L. Ginsburg, F. Maubert \& M. Telles Antunes - Découverte d'Hispanotherium et de Gaindatherium (Rhinocerotidae, Mammalia) dans le Miocène de France. Bull. Mus. natn. Hist. nat., Paris, 4e sér., 9, 1987, section C, nº 3, p. 303-311, 1 est.

\section{9}

M. Telles Antunes \& L. Ginsburg - Les Crocodiliens des faluns miocènes de 1'Anjou. Bull. Mus. natn. Hist. nat., Paris, $4^{\mathrm{e}}$ sér., 11, 1989, section C, nº 2, p. 79-99, 3 est.

\section{2}

Miguel Telles Antunes - Contributions to the Eocene Palaeontology and Stratigraphy of Beira Alta, Portugal. II- New late Eocene mammalian remnants from Côja, Portugal and the presence of Palaeotherium magnum Cuvier. Ciências da Terra (UNL), Lisboa, $n^{\circ} 11$, p.83-89, 1 tab. [P. 89: Remerciements à L. Ginsburg ...].

\section{4}

M. Telles Antunes, B. Azanza, J. Morales \& D. Soria. Los Rumiantes (Artiodactyla, Mammalia) del Mioceno inferior del área de Lisboa (Cuenca del Tajo, Portugal). Rev. Española de Paleontología, 9 (1), pp.51-71, 8 figs., 4 est. [P. 70: Al Dr. Leonard Ginsburg (Paris) por su experto asesoriamiento ...]. 


\section{5}

Léonard Ginsburg \& Miguel Telles Antunes - Les Carnivores du Miocène de Lisbonne (Portugal). Annales de Paléontologie, vol.81, 1995, fasc.3, pp.125-165, 2 quadros, 3 est.

Miguel Telles Antunes - On the Eocene Equid (Mammalia) from Feligueira Grande, Portugal, Paranchilophus lusitanicus (Ginsburg, 1965)/ Taxonomic status, Stratigraphic and Paleogeographical meaning. Comun. Inst. Geol. e Mineiro, 1995 t.81, 1995, pp. 57 - 72. [P. 58-59: «... I asked L. Ginsburg to reopen the Anchilophus lusitanicus case. ... after much comparison work, Ginsburg let me know that he could not proceed further...].

\section{0}

Miguel Telles Antunes - Miocene mammals from Lisbon and geologic age/ A showcase for marine-continental correlations. Ciências da Terra (UNL), Lisboa, $\mathrm{N}^{\circ}$ 14: 343-348, 3 Tab. [P. 343: «The list ... has been made possible owing to ... Ginsburg, ...»].

\section{3}

M. Telles Antunes \& L. Ginsburg - The last Anthracothere Brachyodus onoideus (Mammalia, Artiodactyla) from westernmost Europe and its extinction. Ciências da Terra (UNL), Lisboa, 15, pp.161-172, 2 Tab., 2 Pl.

M. Telles Antunes \& L. Ginsburg - The Deinotherium (Proboscidea, Mammalia): an abnormal tusk from Lisbon, the Miocene record in Portugal and the first appearance datum. Evidence from Lisbon, Portugal. Ciências da Terra (UNL), Lisboa, 15, pp.173-190, 3 Fig., 2 Tab., 4 Pl.

\section{6}

M. Telles Antunes, Balbino, Ausenda C. Balbino \& L. Ginsburg - Miocene Mammalian footprints in coprolites from Lisbon, Portugal. Annales de Paléontologie, (Janvier-Mars 2006), Vol. 92, pp.13-30.

M. Telles Antunes, Ausenda C. Balbino \& L. Ginsburg Ichnological evidence of a Miocene rhinoceros bitten by a beardog (Amphicyon giganteus). Annales de Paléontologie (Janvier-Mars 2006), Vol. 92, pp. 31-39.

Les articles parus en 2006 sont peut-être les dernières publications de L. Ginsburg.

\section{Epilogue}

Ayant dû quitter le Muséum et, en conséquence, perdu les moyens indispensables, le travail scien- tifique de Léonard Ginsburg a cessé. Il ne lui restait plus en dehors de la lecture que d'aller « boire un pot » au bistrot le plus proche, Rue de Rivoli.

Après son départ du Muséum et avant son installation dans une maison de retraite, nous nous rencontrâmes le Dimanche 12 Juin 2005 ! C'était l' « Ami de toujours » mais sensiblement 'diminué', surtout en ce qui concernait la mémoire. Compagnons de carrière, de travail, amis depuis longtemps, nous évoquámes des épisodes du passé et essayâmes de dresser un bilan de nos activités communes. Il m'apprit d'une façon presque indifférente qu'il avait été cambriolé, sa précieuse collection de plats hispano-arabes, turcs et français ayant disparu. Puis, nous nous séparâmes. Je le vis reprendre le chemin de son domicile. Il avait défendu qu'on l'accompagnât et marchait avec difficulté, s'éloignant peu à peu le long du Jardin des Tuileries en direction du Louvre ... ce fut la dernière image que j'emportai de lui ! J'ai appris, par la suite, qu'il avait été admis dans une maison de repos. On disait qu'il ne contrôlait plus ses dépenses...

On peut conclure, sans exagération, qu'on lui doit, entr'autres maintes choses, une contribution fondamentale à la Paléontologie et à la Stratigraphie du Tertiaire du Portugal, ce qui a justifié notamment son élection comme Correspondant Étranger à l'Académie des Sciences de Lisbonne.

En souvenir de son œuvre, de sa collaboration, de son amitié, je tiens à redire à mon excellent ami Léonard ma profonde gratitude et ma sympathie.

Notre Ami commun, Philippe Taquet, a eu la courtoisie de me faire savoir de son décès et de déposer sur sa tombe une fleur à mon intention.

Qu'il repose en paix.

\section{REMERCIEMENTS}

La refaite du texte est due à Mr. Jean-Sébastien Béreau; certains renseignements sont dus à notre cher Collègue, Philippe Taquet. Je leur présente ici l'expression de ma reconnaissance. 